\title{
Tres Métodos en Espectroscopía de Gases para la Obtención de Espectros de Emisión y la Medición de la Constante de Rydberg
}

\author{
RoBerto Mejía ${ }^{1}$ And Jocsan HeRnÁndez ${ }^{1}$ \\ ${ }^{1}$ Universidad Nacional Autónoma de Honduras, mail: roberto.mejia@unah.edu.hn
}

Recibido: 10 de Marzo de 2015 / Aceptado: 4 de Mayo de 2015

\begin{abstract}
Resumen
This article intends to show three methods in gases spectroscopy for wavelengths of an emission spectrum of the hydrogen atom a ranging precision between $0.19 \%$ and $0.95 \%$, and an accuracy between $4.6 \%$ and $0.45 \%$; in the first two methods reference lines defined for two noble gases and mercury vapor are used, which allows adequate calibration for Bunsen-Kirchhoff spectrometer and a configuration using a diffraction replica of 500 lines $/ \mathrm{mm}$ for a first pattern of a not symmetric pattern, in the third method electromagnetic diffraction theory of wavelength is used to determine wavelengths emission of each spectral line observed in a first order symmetric diffraction pattern. Subsequently the validity of more precise and accurate method being verified measure out of the Rydberg constant for three lines of emission in hydrogen with a precision of $0.34 \%$.
\end{abstract}

Keywords: Emission, Wavelength, Hydrogen, Rydberg constant

El presente artículo pretende exponer tres métodos en espectroscopía de gases para obtener las longitudes de onda en el espectro discreto de emisión del hidrógeno con una precisión que oscila entre 0.19\% y $0.95 \%$, y una exactitud entre $4.6 \%$ y $0.45 \%$; en los dos primeros métodos se utilizan como referencia lineas definidas para dos gases nobles y vapor de mercurio, lo que permite una calibración adecuada para un espectómetro Kirchhoff-Bunsen y una configuración utilizando una réplica de difracción de 500 lineas/mm analizando un primer patrón de difracción no simétrico. El tercer método utiliza la teoría de difracción de ondas para determinar la longitud de onda de cada linea espectral de emisión observada en un patron simétrico de primer orden. Posteriormente se verifica la validez del método más exacto y preciso llevando a cabo la medición de la constante de Rydberg para tres lineas de emisión en el hidrógeno con una precisión del $0.34 \%$.

Palabras clave: Emisión, Longitud de Onda, Hidrógeno, Constante de Rydberg

\section{INTRODUCCIÓN}

$\mathrm{H}^{\mathrm{L}}$ L análisis de líneas espectrales de emisión se ha llevado a cabo desde los comienzos del siglo XIX con J. Herschel y F. Talbot (1820) al desarrollar la espectroscopia de emisión de llama con ciertos minerales, posteriormente las investigaciones de J. N. L. Foucault, A. Angström y J. Balmer provocaron una serie de publicaciones que ponen en evidencia los espectros característicos de emisión y absorción de los elementos. En 1860 las contribuciones de Kirchhoff y Bunsen marcaron los inicios de la espectroscopia analítica, pero no fue sino hasta 1913 cuando Niels Bohr [1 ] estableció los cimientos de la mecánica cuántica estableciendo una explicación descriptiva de los espectros atómicos mediante su revolucionario modelo atómico.

Hoy en día la medición y análisis del espectro visible del hidrógeno es uno de los típicos experimentos en un curso de laboratorio avanzado a nivel de pregrado en donde se miden las cuatro líneas visibles para la serie de Balmer incluyendo la verificación de la formula de Balmer y la medición de la constante de Rydberg. Por razones históricas se considera la espectroscopia del hidrógeno una de las piezas claves en el desarrollo de la mecánica cuántica; sin embargo el espectro de otros átomos no es simple de analizar, simplemente considerar más de un protón y un electrón para un átomo como el caso del helio implica un mayor número de grados de libertad que hace que el problema sea muy complejo, en ese sentido un curso de pregrado no basta para entender los niveles de energía del helio o mercurio así como fermiones idénticos [2 ]. En el caso de espectros más complejos como el del Helio el experimento se limita a medir las longitudes de onda emitidas utilizando valores tabulados para identificar las transiciones atómicas correspondientes [3 ], sin embargo estos experimentos introducen a los estudiantes en la terminología utilizada en física atómica mediante un análisis cuantitativo y cualitativo de los niveles de energía atómicos cuantizados.

En este artículo se presentan tres métodos experimentales muy simples para la medición de las longitudes de onda de un espectro de emisión, lo que permite establecer 
la metodología más adecuada en función de la exactitud y precisión que se requiere; finalmente se utiliza el método más preciso para llevar a cabo la medición de la constante de Rydberg. Para las dos primeras configuraciones, antes de llevar a cabo el experimento con el espectro del hidrógeno, se lleva a cabo una calibración utilizando como referencia fuentes de emisión estándar, en nuestro caso: helio, kriptón y vapor de mercurio; la tercera configuración utiliza el principio de difracción de un haz compuesto para un patrón simétrico. Adicionalmente se pretende exponer el desarrollo de dos experimentos en su versión mejorada a los realizados en la Escuela de Física UNAH $[4]$.

\section{CONFIguración EXPERIMENTAL}

\section{A. El Espectrómetro Kirchhoff-Bunsen}

Aparato desarrollado por G. Kirchhoff y R. Bunsen en 1859 para determinar espectros característicos de emisión en ciertos elementos [5 ]. El dispositivo está compuesto por un colimador en donde se enfoca el haz de emisión en un prisma ubicado en el centro del espectrómetro, una escala definida por otro colimador, y un telescopio en donde se observan los haces monocromáticos refractados por el prisma.

El primer método expuesto utiliza un espectrómetro Kirchhoff-Bunsen, el funcionamiento simple para la configuración utilizada en el laboratorio se muestra en la figura 1, en este caso se ha colocado una cámara digital de 12.0 MP para efectuar las lecturas de la posición de cada línea espectral en la escala del espectrómetro. La figura 2 muestra el espectrómetro utilizado en el laboratorio, utilizando una de las dos fuentes de alta tensión AC/DC con diferencias de potencial mayores a $1.0 \mathrm{kV}$ lo que permite ionizar el gas contenido en el tubo espectral el que se encuentra instalado dentro de una pequeña cámara obscura que evita la contaminación luminosa; por otro lado se utiliza una fuente incandescente para iluminar la escala del espectrómetro.

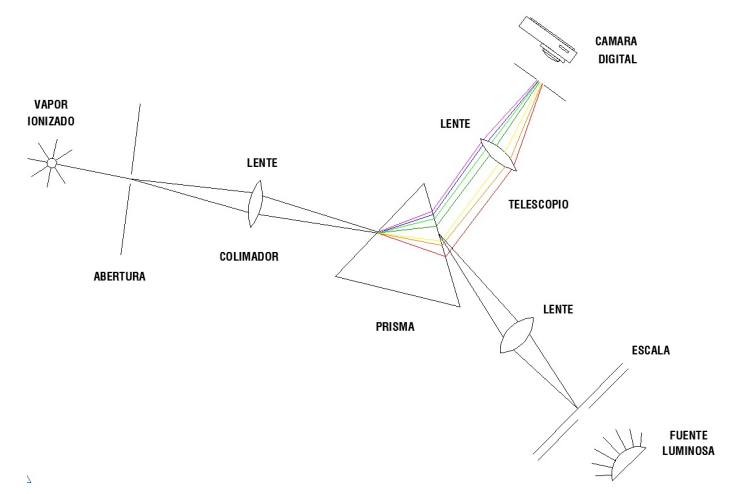

Figura 1: Diagrama de configuración utilizado para el espectrómetro Kirchhoff-Bunsen

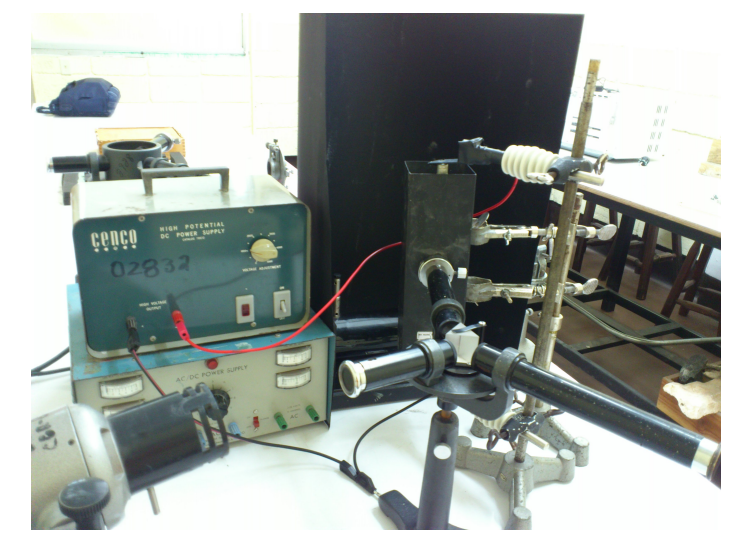

Figura 2: Montaje experimental utilizado en el laboratorio con el espectrómetro Kirchhoff-Bunsen

B. Patrón de difracción no simétrico de un haz policromático

El segundo método utiliza una configuración como la mostrada en la figura 3. Utilizando un patrón lateral de difracción no simétrico se permite realizar una calibración en base a la relación entre la posición lineal de cada línea espectral con la posición horizontal correspondiente; la figura 4 muestra el montaje para esta configuración. Este principio es utilizado en el espectrómetro de difracción [5 ] [6 ] [7 ].

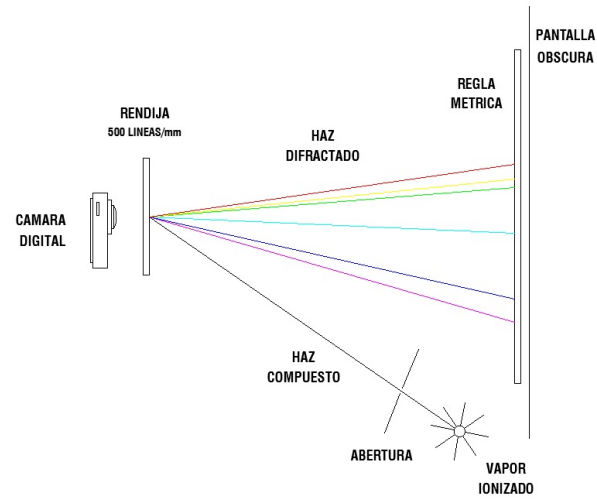

Figura 3: Diagrama de configuración utilizado para la difracción del haz policromático por emisión de la fuente de referencia

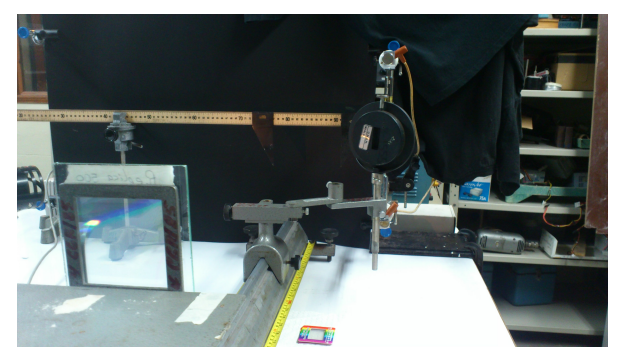

Figura 4: Montaje experimental con la configuración para la difracción no simétrica del haz policromático emitido por la fuente espectral 
Para el tercer método se hace uso de un patrón simétrico difractado sobre el cual se obtienen las longitudes de onda correspondientes a las líneas de emisión observadas simplemente haciendo uso del principio de difracción mediante la explicación ondulatoria de la luz. En la figura 5 se muestra un esquema de esta tercera configuración y en la figura 6 se muestra el montaje utilizado en el laboratorio.

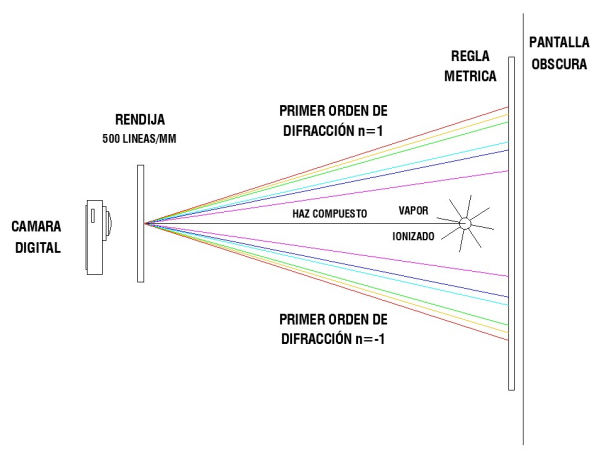

Figura 5: Diagrama de configuración utilizado para la difracción del haz policromático por emisión de la fuente de referencia

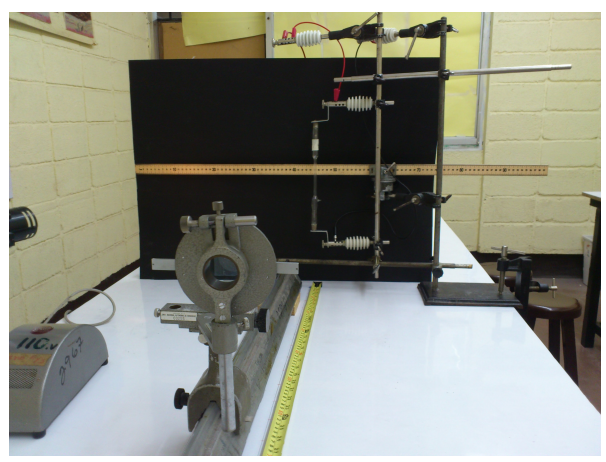

Figura 6: Montaje experimental para la configuración de difracción para un patrón no simétrico

\section{Calibración}

La calibración del espectrómetro Kirchhoff-Bunsen utilizado en el laboratorio se llevo a cabo considerando dos modelos empíricos para el ajuste de datos. El primer modelo a considerar fue el de Cauchy [5 ] [8 ] y el segundo es el de Hartmann [5 ] [8 ]. Ambos modelos empíricos se ajustan adecuadamente para la relación entre la posición horizontal de cada línea espectral y la longitud de onda correspondiente vista en la escala del espectrómetro, las figuras 7, 8 y 9 muestran los espectros observados con la primera configuración. La comparación entre los dos modelos en base a lo observado para el helio, kryptón y vapor de mercurio se expone en la figura 10

El modelo de ajuste de Cauchy para el espectrómetro utilizado corresponde a la ecuación:

$$
x(\lambda)=A+\frac{B}{\lambda^{2}}+\frac{C}{\lambda^{4}}+\ldots
$$

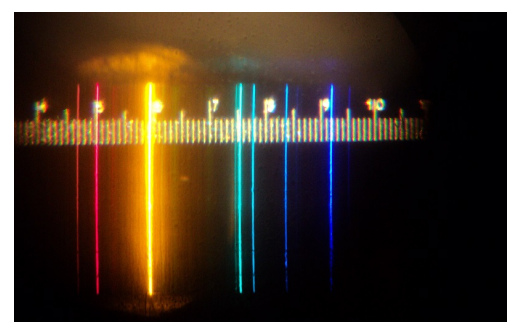

Figura 7: Espectro del Helio observado en el espectrómetro Kirchhoff-Bunsen

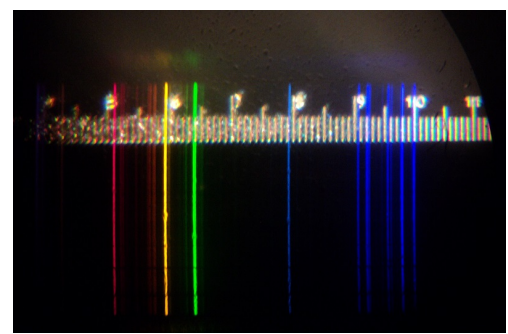

Figura 8: Espectro del Kriptón observado en el espectrómetro Kirchhoff-Bunsen

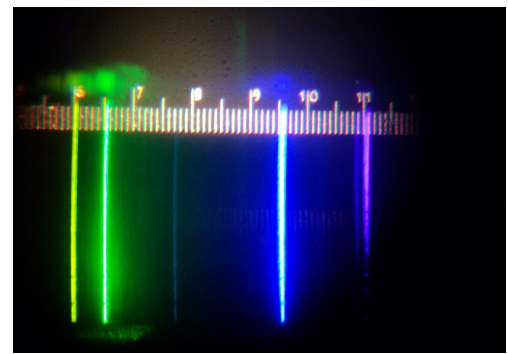

Figura 9: Espectro del vapor de Mercurio observado en el espectrómetro Kirchhoff-Bunsen

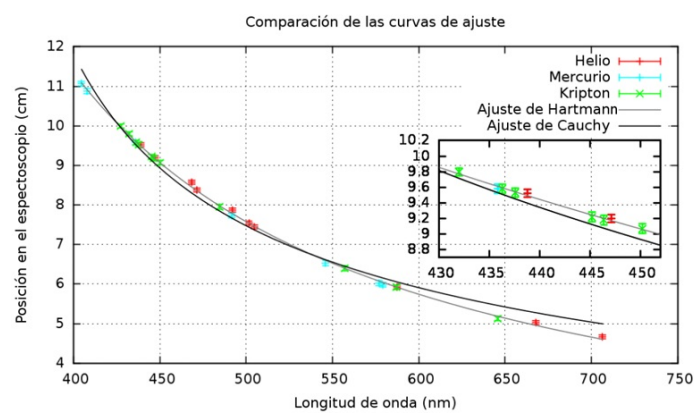

Figura 10: Relación entre la posición en la escala de espectrómetro con cada una de las 28 longitudes de onda de referencia obtenidas del Helio, Kriptón y Mercurio

En donde solo se consideraron las constantes A y B El modelo de Hartmann corresponde a la ecuación:

$$
x(\lambda)=A+\frac{B}{C-\lambda}
$$

En casos más generales resulta más conveniente utilizar el modelo de Sellmeier [8 ] pero para nuestro caso el modelo de Hartmann resulta suficiente. 
La tabla 1 presenta los valores medidos de la posición horizontal para cada longitud de onda emitida. Se lograron registrar como referencia 10 lineas para el espectro del Helio, 10 lineas para el Kriptón y 8 lineas para el Mercurio; las longitudes de onda de estas lineas de referencias fueron obtenidas del Handbook of Basic Atomic Spectroscopic Database, en el Physical Measurement Laboratory, NIST [12 ] y la base de datos del observatorio astronómico de la universidad de Strasbourg [14] para un total de 28 datos de referencia para la calibración del espectrómetro. Con la ecuación de calibración es posible efectuar las mediciones de la longitud de onda en nanómetros para las lineas de emisión del Hidrógeno

$$
\lambda(x)=211.0+\frac{2060}{x-0.44}
$$

\begin{tabular}{|c|c|c|c|c|c|c|}
\hline Elemento & Color & $\lambda(\mathrm{nm})$ & $\bar{x}(\mathrm{~cm})$ & \multicolumn{3}{|c|}{ Hartman } \\
\hline \multirow{10}{*}{ Helio } & Rojo 1 & 706.5171 & 4.66 & $A(\mathrm{~cm})$ & $B(\mathrm{~cm} \cdot \mathrm{nm})$ & $C(\mathrm{~nm})$ \\
\hline & Rojo 2 & 667.81517 & 5.02 & \multirow{4}{*}{$0.44 \pm 0.16$} & \multirow{4}{*}{$-2060 \pm 90$} & \multirow{4}{*}{$211 \pm 6$} \\
\hline & Amarillo & 587.56148 & 5.9 & & & \\
\hline & Verde 1 & 504.774 & 7.45 & & & \\
\hline & Verde 2 & 501.5678 & 7.55 & & & \\
\hline & Aqua 1 & 492.1931 & 7.87 & \multicolumn{2}{|c|}{ Cauchy } & \\
\hline & Aqua 2 & 471.3146 & 8.37 & $A(\mathrm{~nm})$ & $B\left(\mathrm{~cm}^{2} \cdot \mathrm{nm}\right)$ & \\
\hline & Aqua 3 & 468.53769 & 5.57 & \multirow{3}{*}{$333 \pm 3$} & \multirow{3}{*}{$9300 \pm 200$} & \\
\hline & Azul & 447.1479 & 9.20 & & & \\
\hline & Violeta & 438.79289 & 9.52 & & & \\
\hline \multirow{7}{*}{ Mercurio } & Amarillo1* & 579.1 & 5.98 & & & \\
\hline & Amarillo2* & 577.0 & 6.02 & & & \\
\hline & Verde & 546.0735 & 6.53 & & & \\
\hline & Azul * & 491.6 & 7.73 & & & \\
\hline & Violeta 1 & 435.8328 & 9.08 & & & \\
\hline & Violeta $2^{*}$ & 407.8 & 10.88 & & & \\
\hline & Violeta 3 & 404.6563 & 11.07 & & & \\
\hline \multirow{11}{*}{ Kriptón } & Rojo & 642.018 & 5.13 & & & \\
\hline & Amarillo & 587.09160 & 5.92 & & & \\
\hline & Verde & 557.0289 & 6.40 & & & \\
\hline & Aqua & 484.6612 & 7.92 & & & \\
\hline & Violeta $1^{*}$ & 450.2 & 9.07 & & & \\
\hline & Violeta $2^{*}$ & 446.4 & 9.18 & & & \\
\hline & Violeta $3 *$ & 445.2 & 9.22 & & & \\
\hline & Violeta $4^{*}$ & 437.6 & 9.53 & & & \\
\hline & Violeta $5^{*}$ & 437.6 & 9.58 & & & \\
\hline & Violeta $6^{*}$ & 436.3 & 9.80 & & & \\
\hline & Violeta $7^{*}$ & 427.4 & 10.00 & & & \\
\hline
\end{tabular}

Tabla 1: Valores de las longitudes de onda encontradas para Helio, Kriptón y Mercurio, posición en el espectroscopio y constantes para las curvas de ajuste.

Donde:

Elemento: elemento utilizado.

Color: color visto en el espectrómetro.

$\lambda$ : longitud de onda del haz según [12 ].

$\bar{x}$ : mejor valor medido para la posición horizontal en la escala de espectrómetro.

Constantes según la ecuación de Hartmann: $\bar{x}=A+B /\left(C-\lambda_{\text {ref }}\right)$.

Constantes según el ajuste de Cauchy: $\bar{x}=A+B / \lambda_{\text {ref }}^{2}$.

*: Longitud de onda según [14]. 


\begin{tabular}{|c|c|c|c|c|}
\hline Elemento & Color & $\lambda(\mathrm{nm})$ & $\bar{x}(\mathrm{~cm})$ & $\lambda^{\prime}(\mathrm{nm})$ \\
\hline \multirow{4}{*}{ Hidrógeno } & Rojo & 656.2711 & 5.12 & $650 \pm 30$ \\
\cline { 2 - 5 } & Aqua & 486.13615 & 7.93 & $486 \pm 14$ \\
\cline { 2 - 5 } & Violeta 1 & 434.0462 & 9.73 & $432 \pm 12$ \\
\cline { 2 - 5 } & Violeta 2 & 410.174 & 10.83 & $409 \pm 11$ \\
\hline
\end{tabular}

Tabla 2: Longitudes de onda de las lineas espectrales de emisión para el Hidrógeno utilizando la ecuación de calibración.

Donde:

Elemento: Elemento usado para ver las líneas del espectro.

Color: color observado en el espectroscopio.

$\lambda$ : Longitud de onda según [12 ].

$\bar{x}$ : Mejor valor medido para la posición horizontal en la escala del espectrómetro.

$\lambda^{\prime}$ : Longitud de onda media según la ecuación de calibración.

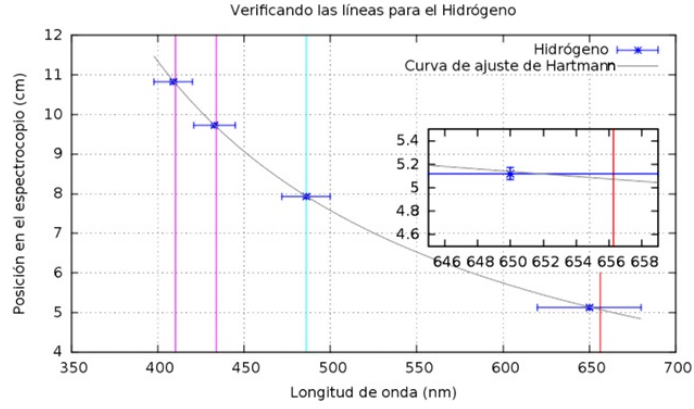

Figura 11: Líneas esperadas para el hidrógeno

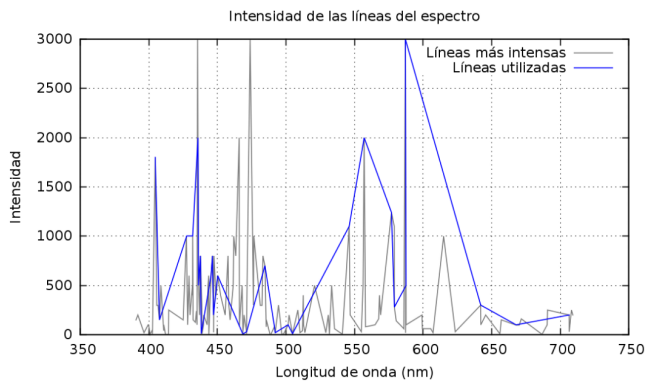

Figura 12: Longitudes de onda con su intensidad

Para el segundo método se hace uso del principio de difracción mediante el cual es posible verificar la relación entre la posición horizontal de las lineas espectrales del primer patrón de difracción observado y la longitud de onda de cada linea, para el caso de $d=2.0 \mu \mathrm{m}$ y $\lambda=$ [400 nm, $700 \mathrm{~nm}$ ] la relación es casi lineal, por lo que un modelo lineal para este rango visible permite una adecuada calibración

$$
\begin{gathered}
Y_{n}=\frac{L}{\sqrt{\left(\frac{d}{n \lambda}\right)^{2}-1}} \\
x(\lambda)=A \lambda+B
\end{gathered}
$$

$Y_{n}$ Corresponde a la posición horizontal del patrón de difracción de orden $n$ para un haz que atravieza una

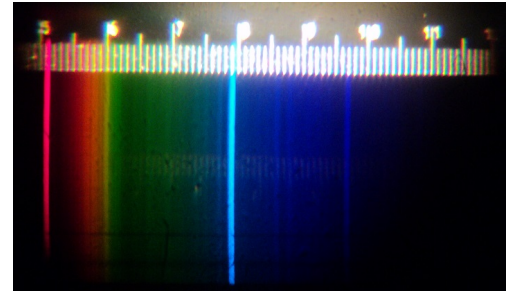

Figura 13: Espectro del Hidrógeno observado en el espectrómetro Kirchhoff-Bunsen

rendija multiple con separación $d$ entre aberturas, y proyectadas a una distancia $L$ de la rendija. La figura 14 muestra la configuración del experimento utilizando un tubo espectral con Kriptón, y las figuras 15, 16 y 17 muestran los espectros observados con esta segunda confi-

\begin{tabular}{|c|c|c|c|c|c|}
\hline Elem & Color & $\lambda(\mathrm{nm})$ & $\bar{x}(\mathrm{~cm})$ & $A$ & $B(\mathrm{~cm})$ \\
\hline \multirow{7}{*}{$\mathrm{Hg}$} & Verde1* & 579.1 & 82.78 & \multirow{24}{*}{$\begin{array}{r}-0.0374 \\
\pm 0.0002\end{array}$} & \multirow{24}{*}{$\begin{array}{l}104.55 \\
\pm 0.08\end{array}$} \\
\hline & Verde2* & 577.0 & 83.83 & & \\
\hline & Verde3 & 546.074 & 83.97 & & \\
\hline & Aqua1 & 520.477 & 85.13 & & \\
\hline & Aqua2* & 491.6 & 86.08 & & \\
\hline & Azul & 435.833 & 88.18 & & \\
\hline & Violeta* & 407.8 & 89.37 & & \\
\hline \multirow{9}{*}{$\mathrm{Kr}$} & Roja & 657.007 & 80.08 & & \\
\hline & Amarilla & 587.092 & 82.13 & & \\
\hline & Verde & 557.029 & 83.77 & & \\
\hline & Azul & 484.661 & 86.38 & & \\
\hline & Violeta1* & 450.2 & 87.72 & & \\
\hline & Violeta2* & 445.2 & 87.92 & & \\
\hline & Violeta $3^{*}$ & 436.3 & 88.22 & & \\
\hline & Violeta4* & 432.0 & 88.42 & & \\
\hline & Violeta5* & 427.4 & 88.62 & & \\
\hline \multirow{8}{*}{$\mathrm{He}$} & Roja 1 & 706.51771 & 78.17 & & \\
\hline & Roja 2 & 667.81517 & 79.58 & & \\
\hline & Amarilla & 587.56148 & 82.67 & & \\
\hline & Verde & 501.5678 & 85.82 & & \\
\hline & Aqua 1 & 492.1931 & 86.13 & & \\
\hline & Aqua 2 & 471.3146 & 86.97 & & \\
\hline & Azul & 447.1479 & 87.82 & & \\
\hline & Violeta & 388.6489 & 90.02 & & \\
\hline
\end{tabular}
guración.

Tabla 3: Longitudes de onda de referencia para Helio, 
Mercurio y Kriptón. Resultado del ajuste lineal que relaciona estas longitudes de onda con la posición horizontal para una configuración fija

Donde:

Elemento: elemento utilizado.

Color: color visto en la experiencia.

$\lambda$ : longitud de onda del haz según [12 ].

$\bar{x}$ : mejor valor medido para la posición.

Constantes de la recta de ajuste: $x=\lambda A+B$

*: longitud de onda del haz según [14 ].

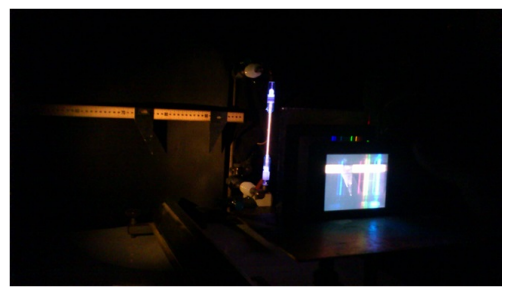

Figura 14: Configuración experimental utilizada en el laboratorio

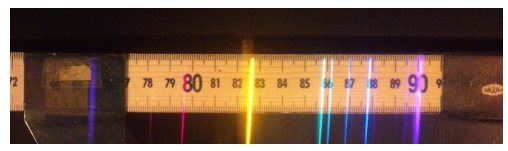

Figura 15: Espectro del Helio observado en la réplica de difracción

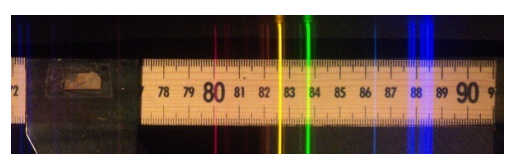

Figura 16: Espectro del Kriptón observado en la réplica de difracción

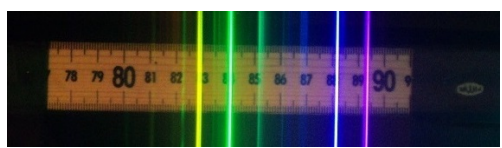

Figura 17: Espectro del Mercurio observado en la réplica de difracción

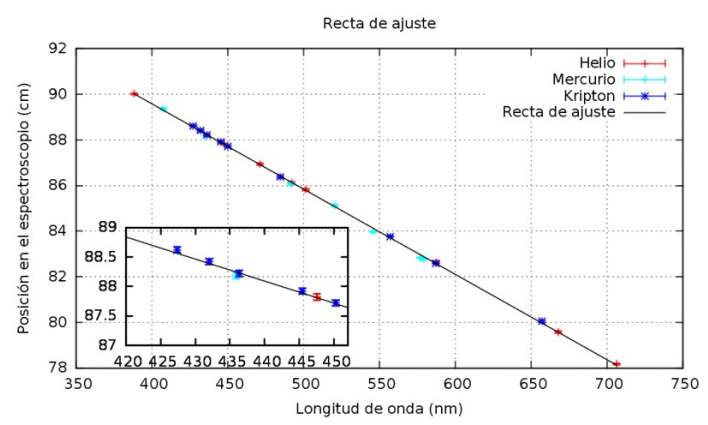

Figura 18: Ajuste lineal de la relación entre la posición de cada linea con su longitud de onda aceptada
Con la ecuación de calibración es posible efectuar las mediciones de la longitud de onda en nanómetros para las lineas de emisión del hidrógeno, para el valor de $x$ en centímetros

$$
\lambda(x)=-26.73 x+2795.45
$$

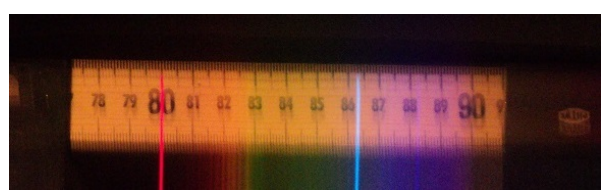

Figura 19: Espectro del Hidrógeno observado en la réplica de difracción

Ahora y según la tabla 4, se puede observar que los resultados son muy buenos, excepto para la línea espectral roja (esto se ha vuelto recurrente y puede deberse a contaminaciones en el tubo espectral), sin embargo con las barras de error el resultado se vuelve aceptable.

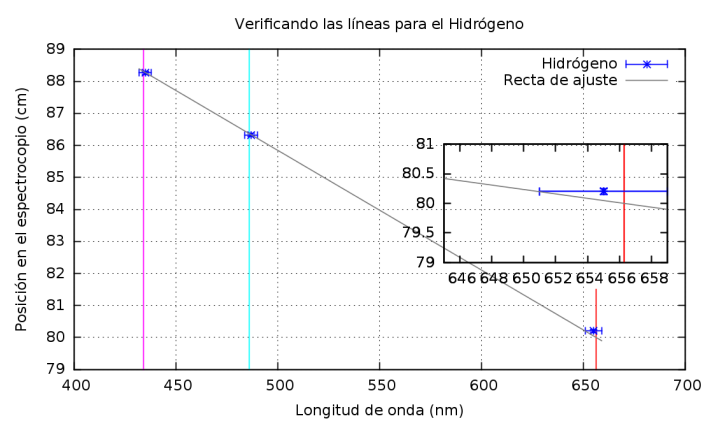

Figura 20: Verificando el comportamiento para el ajuste del hidrógeno

\begin{tabular}{|c|c|c|c|c|}
\hline $\mathrm{E}$ & Color & $\lambda(\mathrm{nm})$ & $\bar{x}(\mathrm{~cm})$ & $\lambda^{\prime}(\mathrm{nm})$ \\
\hline \multirow{3}{*}{$\mathrm{H}$} & Roja & 656.2711 & 80.02 & $655 \pm 4$ \\
\cline { 2 - 5 } & Azul & 486.13615 & 86.32 & $487 \pm 3$ \\
\cline { 2 - 5 } & Violeta & 434.0462 & 88.27 & $435 \pm 3$ \\
\hline
\end{tabular}

Tabla 4: Valores de las longitudes de onda encontradas para el Hidrógeno según la recta de ajuste

Donde:

E: Elemento utilizado.

Color: Color visto en la experiencia.

$\lambda$ : Longitud de onda del haz según [12 ].

$\bar{x}$ : Mejor valor medido para la posición.

$\lambda^{\prime}$ : Longitud de onda calculada apartir de la ecuación de ajuste $x=A \lambda+B$.

El resultado notable de este método se manifiesta en una mejoría en la precisión y exactitud, la incertidumbre se reduce en un $80 \%$ y se mejora la exactitud en casi $1.0 \%$.

Por último vale considerar las líneas que se usaron en el experimento y el total del espectro, esto para efectos comparativos y además ayuda a determinar si estamos tratando con elementos puros o no. 
Es de hacerse notar que aunque muchas de las líneas coinciden en las dos experiencias, en realidad no todas son iguales, en especial en los extremos, la diferencia principal entre las dos experiencias es lo que se usó para generar la difracción, así que es muy posible que la diferencia en las líneas espectrales se deba a ello.

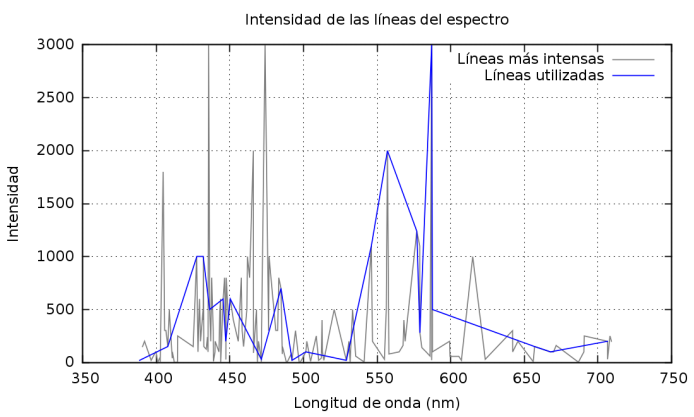

Figura 21: Líneas utilizadas y líneas más intensas del espectro

Para el tercer método se utiliza un patrón central de difracción considerando la ecuación mostrada para $n=1$, se pueden medir las longitudes de onda para el espectro de emisión del hidrógeno:

$$
\lambda=\frac{d / n}{\sqrt{1+\left(L / Y_{n}\right)^{2}}}
$$

Ecuación que permite determinar la longitud de onda de la linea espectral difractada por la réplica de 500 lineas $/ \mathrm{mm}$, separada una distancia $d$ entre aberturas, y proyectadas a una distancia $L$ de la rendija. El patrón de difracción obtenido para efectuar las mediciones es de primer orden $n=1$

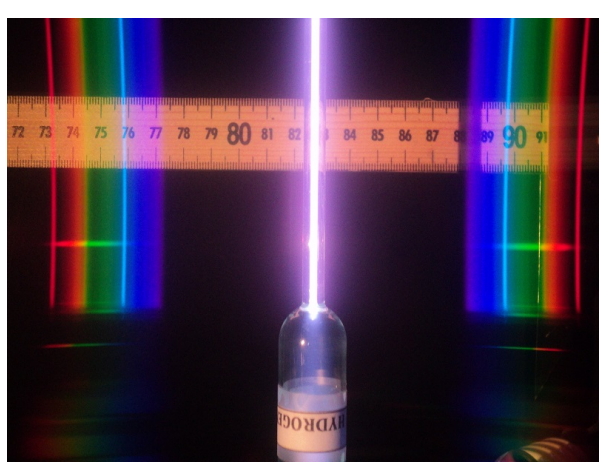

Figura 22: Líneas utilizadas y líneas más intensas del espectro

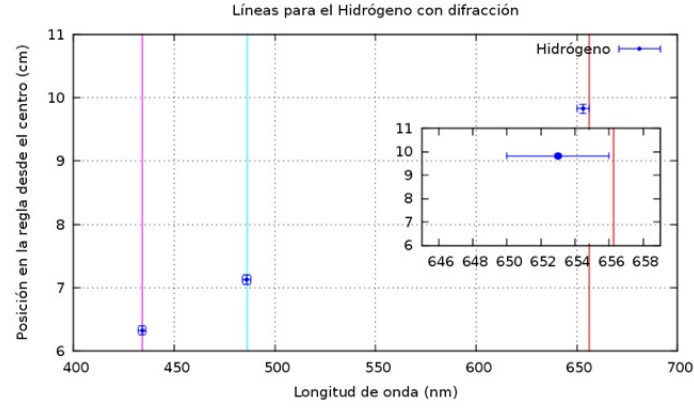

Figura 23: Las tres longitudes de onda mas intensas en el espectro de emisión del Hidrógeno

\begin{tabular}{|c|c|c|}
\hline Color & $\lambda(\mathrm{nm})$ & $\lambda^{\prime}(\mathrm{nm})$ \\
\hline Rojo & 656.2711 & $653 \pm 3$ \\
\hline Aqua & 486.13615 & $486 \pm 2$ \\
\hline Violeta 1 & 434.0462 & $434 \pm 2$ \\
\hline
\end{tabular}

Figura 24: Comparación entre los valores aceptados de las longitudes de onda para el espectro del hidrógeno (NIST), y las longitudes de onda obtenidas utilizando el primer orden de difracción para un patrón simétrico en el espectro de emisión del hidrógeno.

\section{Medición de la constante de Rydberg}

Finalmente utilizando las longitudes de onda obtenidas mediante el primer patrón central de difracción, es posible realizar un ajuste utilizando como modelo la ecuación de Rydberg para el átomo de hidrógeno. Se sabe que la ecuación de Rydberg para el átomo de hidrógeno permite obtener la energía del fotón emitido en una transición,

$$
E_{\gamma}=\Delta E=h c R_{\infty} \frac{1}{n_{f}^{2}}-\left.\frac{h c R_{\infty}}{n_{i}^{2}}\right|_{n_{f}=2}
$$

El caso que se analiza es el espectro de emisión en el rango visible de longitudes de onda debido a las transiciones del primer estado excitado $\left(n_{f}=2\right)$, las que constituyen la serie de Balmer, como se muestra en la figura 25. El modelo utilizado para el ajuste que relaciona la energía del fotón con el número cuántico de la transición,

$$
E_{\gamma}=\Delta E=-h c R_{\infty} \frac{1}{n_{i}^{2}}+\frac{1}{4} h c R_{\infty}=A \frac{1}{n_{i}^{2}}+B
$$

La constante de Rydberg se puede determinar de dos formas utilizando un mismo modelo,

$$
R_{\infty}=-\frac{A}{h c} \quad R_{\infty}=-\frac{4 B}{h c}
$$

Utilizando las longitudes de onda mostradas en la figura 23 , es posible realizar un ajuste de $E_{\gamma}\left(n_{i}\right)$. la siguiente gráfica muestra el resultado de este ajuste 


\begin{tabular}{|c|c|c|c|c|c|c|c|c|c|}
\hline $\mathrm{E}$ & $\mathrm{T}$ & Color & $\lambda(\mathrm{nm})$ & $x(\mathrm{~cm})$ & $\begin{array}{c}\overline{\lambda^{\prime}} \\
(\mathrm{nm})\end{array}$ & $n_{1}$ & $n_{2}$ & $A(\mathrm{eV})$ & $B(\mathrm{eV})$ \\
\hline \multirow{6}{*}{$\mathrm{H}$} & \multirow{3}{*}{1} & Rojo & 656.2711 & 9.85 & 653 & 3 & \multirow{6}{*}{2} & \multirow{2}{*}{$-13.37 \pm 0.03$} & \multirow{2}{*}{$3.388 \pm 0.003$} \\
\hline & & Azul & 486.13615 & 7.15 & 486 & 4 & & & \\
\hline & & Violeta & 434.0462 & 6.35 & 434 & 5 & & \multirow[t]{4}{*}{$R_{A}\left(\mathrm{~m}^{-1}\right)$} & $R_{B}\left(\mathrm{~m}^{-1}\right)$ \\
\hline & \multirow{3}{*}{2} & Rojo & 656.2711 & 9.80 & 651 & 3 & & & \multirow{3}{*}{$\left(1.09 \pm 0.089 \times 10^{7}\right.$} \\
\hline & & Azul & 486.13615 & 7.15 & 486 & 4 & & & \\
\hline & & Violeta & 434.0462 & 6.36 & 435 & 5 & & & \\
\hline
\end{tabular}

Tabla No. 4: Mejores valores obtenidos para las longitudes de onda del Hidrógeno utilizando el tercer método, y resultados del ajuste utilizando un modelo basado en la ecuación de Rydberg para el Hidrógeno.

Donde:

E: $\quad$ Elemento utilizado.

T: $\quad$ Número del tubo espectral del elemento utilizado.

Color: Color observado en la experiencia.

$\lambda: \quad$ Longitud de onda según [12 ].

$x$ : $\quad$ Posición observada desde el centro del tubo (83 $\mathrm{cm}$ de la regla).

$\overline{\lambda^{\prime}}: \quad$ Mejor longitud de onda medida utilizando la ecuación de difracción.

$n_{1}$ : Número cuántico principal para el nivel inicial en la transición del electrón.

$n_{2}$ : Número cuántico principal para el nivel final en la transición del electrón.

Constantes de la curva de ajuste con su respectiva incertidumbre: $\quad A / n_{i}{ }^{2}+B$

$R_{A}$ : Constante de Rydberg utilizando el valor de A del ajuste y su respectiva inscertidumbre.

$R_{B}$ : Constante de Rydberg utilizando el valor de B del ajuste y su respectiva incertidumbre.

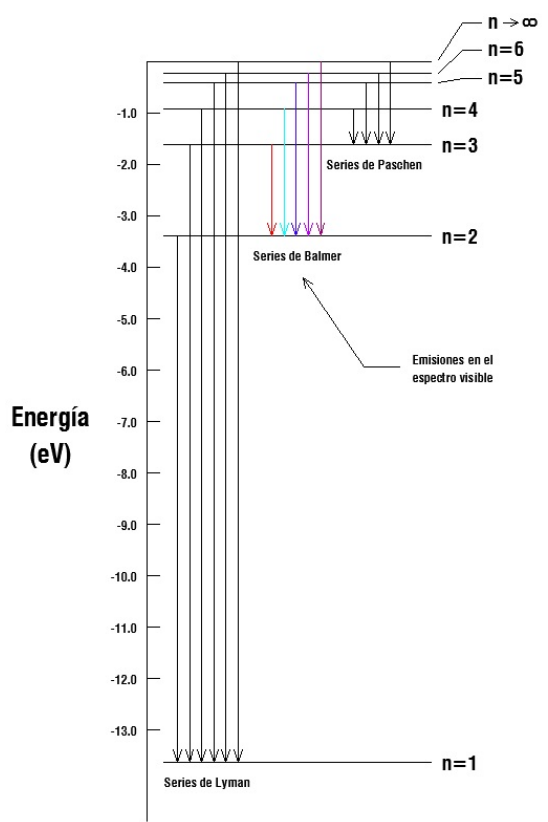

Figura 25: Niveles de energía y transiciones en el espectro del Hidrógeno

Se puede apreciar que ambos valores, para ambos métodos resultaron muy cercanos al teórico, aunque utilizando el coeficiente B se obtiene una mayor presición, el valor aceptado para la constante de Rydberg es de $10973731.568549 \mathrm{~m}^{-1}[\mathbf{1 6}]$

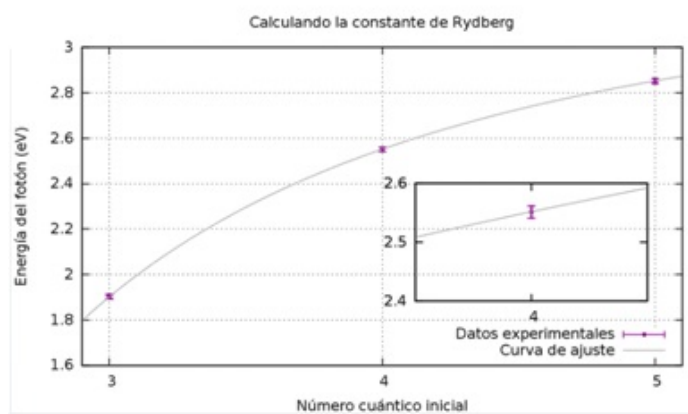

Figura 26: Curva de ajuste para el modelo de Rydberg utilizado en la serie de Balmer

\section{Conclusiones}

- El modelo de Hartmann permite un mejor ajuste de datos que el modelo de Cauchy, ya que se basa en la ecuación de Sellmeier que relaciona empíricamente el indice de refracción con la longitud de onda para un medio transparente particular, como es el caso del prisma instalado en el espectrómetro KirchhoffBunsen

- La configuración experimental con la rendija de difracción permite mejorar la obtención de las longitudes de onda en un $90 \%$ ya que una adecuada calibración permite incertidumbres porcentuales entre $0.5 \%$ y $1.5 \%$

- Utilizando un primer patrón simétrico de difracción para el haz policromático de emisión en el hidrógeno es posible obtener longitudes de onda en el espectro 
visible con una presición del $99.54 \%$ y reduciendo la exactitud a un $0.45 \%$ permitiendo medir la constante de Rydberg con una exactitud del $99.32 \%$ al valor aceptado $[\mathbf{1 6}]$

\section{REFERENCIAS}

[1] URL: http://physics .nist . gov/PhysRefData/ Handbook\%5C\%5C/element_\%7B-\%7Dname.htm.

[2] URL://astro.u-strasbg.fr/koppen/discharge/ index.html.

[3] Physics 152. "Diffraction Gratings and the Hydrogen Spectrum". En: Department of Physics. ST. Lawrence University (2004).

[4] V. Bruckner. "To the use of Sellmeier formula". En: Senior Experten Service (SES) Bonn and HfT Leipzig ().

[5] Rydberg Constant. "Fundamental Physical". En: The NIST Reference on Constants, Units and Uncertainty, Physical Measurement Laboratory, NIST ().

[6] R. M. Eisberg. Fundamentos de Física Moderna. Lamisa, México, 1997. Cap. 5.

[7] R. Estey. "The Use of AO Spencer Spectrometer". En: American Optical Co. Instrument Division (1938).
[8] "Experimentos Clásicos en Espectroscopía de Gases Desarrollados en la Carrera de Física de la UNAH". En: Revista de la Escuela de Física II.2 (2014).

[9] R. L. Liboff. Introductory Quantum Mechanics. 3. ${ }^{\mathrm{a}} \mathrm{ed}$. Addison-Wesley, Reading, MA, 1998.

[10] D. Morin. Interferance and Difraction. Departament of Physics, Hardvard University, 2010.

[11] M. C. Lo Presto. "A closer look at the spectrum of helium". En: Phys. Teach 36 (1998), págs. 172-173.

[12] AO Spencer R. Estey. "The use of the spectrometer". En: Research Physicist, American Optical Co. (1938).

[13] c. R. Nave. "Hydrogen Spectrum and Energy". En: Quantum Physics, Hyperphysics, Georgia State University (2014).

[14] "Selective experiments in physics: The prism spectrometer". En: ().

[15] The Hydrogen Spectrum. "Chemestry 12 General Chemestry". En: Department of Physical Science Kingsborough Community College The City University of New York (Winter 2010).

[16] J. R. Taylor. Classical Mechanics. University Science Books, U.S.A, 2005. 